\title{
A Tensor-Based Morphometry Study of Genetic Influences on Brain Structure Using a New Fluid Registration Method
}

\author{
Caroline Brun ${ }^{1}$, Natasha Leporé ${ }^{1}$, Xavier Pennec ${ }^{2}$, Yi-Yu Chou ${ }^{1}$, \\ Agatha D. Lee ${ }^{1}$, Marina Barysheva ${ }^{1}$, Grieg de Zubicaray ${ }^{3}$, \\ Matthew Meredith ${ }^{3}$, Katie McMahon ${ }^{3}$, Margaret J. Wright ${ }^{4}$, \\ Arthur W. Toga ${ }^{1}$, and Paul M. Thompson ${ }^{1}$ \\ ${ }^{1}$ Laboratory of Neuro Imaging, UCLA, Los Angeles, CA 90095, USA \\ ${ }^{2}$ Asclepios Research Project, INRIA, 06902 Sophia-Antipolis Cedex, France \\ ${ }^{3}$ Centre for Magnetic Resonance, University of Queensland, Brisbane, Australia \\ ${ }^{4}$ Genetic Epidemiology Lab, QIMR, Brisbane, Australia
}

\begin{abstract}
We incorporated a new Riemannian fluid registration algorithm into a general MRI analysis method called tensor-based morphometry to map the heritability of brain morphology in MR images from 23 monozygotic and 23 dizygotic twin pairs. All 92 3D scans were fluidly registered to a common template. Voxelwise Jacobian determinants were computed from the deformation fields to assess local volumetric differences across subjects. Heritability maps were computed from the intraclass correlations and their significance was assessed using voxelwise permutation tests. Lobar volume heritability was also studied using the ACE genetic model. The performance of this Riemannian algorithm was compared to a more standard fluid registration algorithm: 3D maps from both registration techniques displayed similar heritability patterns throughout the brain. Power improvements were quantified by comparing the cumulative distribution functions of the $p$-values generated from both competing methods. The Riemannian algorithm outperformed the standard fluid registration.
\end{abstract}

\section{Introduction}

Nonlinear registration is of particular importance in medical imaging, as it can be used to localize regions of anatomical change in longitudinal studies and drug trials, as well as group differences in brain structure. In particular, non rigid warping is the first step in many brain structural analyses, such as TensorBased Morphometry (TBM) 2] 9], a popular method which is increasingly used to detect morphometric differences associated with disease [16] 6], normal and abnormal development 27] and cognitive performance.

In TBM, two groups of images are compared, and statistical differences in local shape and volume are determined. TBM starts with a linear registration of a set of brain images to a common space. All scans are then nonlinearly registered to a common template, which is typically either a single individual

D. Metaxas et al. (Eds.): MICCAI 2008, Part II, LNCS 5242, pp. 914 -921 2008.

(C) Springer-Verlag Berlin Heidelberg 2008 
in the study or a group average template [15]. Images are driven to similarity using common landmarks, or measures over the whole image such as the squaredintensity difference $\left(L^{2}\right.$-norm), cross-correlation or more complex metrics that can be derived from information-theory, such as the Jensen-Rényi divergence 6]. The transformation is constrained through a regularizer to enforce desirable properties in the deformation, such as smoothness, invertibility and inverseconsistency 5 .

Amongst the various existing techniques, some registration methods treat the image as an elastic or fluid medium [11, deformed according to mechanical equations. At each voxel, an internal force field, derived from the similarity measure, drives the template image toward the study, increasing anatomical correspondence. In standard methods, this transformation is constrained and regularized by restoring forces according to the Navier-Lamé equation. Statistical information may also be included in the registration process [12] 14. Recently, Pennec in 22] proposed a statistical prior that regularizes the deformation tensors $\Sigma$. Deformation tensors are symmetric-positive definite matrices and do not form a vector space under standard algebraic operations (e.g., matrix addition and scalar multiplication). In Arsigny et al. 1] the matrix logarithm of $\Sigma$ was used to project its value into the tangent space at the origin, and to define a vector space structure on the manifold of positive definite tensors. Pennec et al. proposed the $\log$-Euclidean Riemannian elasticity regularizer $R e g_{R E}$ in this space:

$$
\operatorname{Reg}_{R E}(\Sigma)=\frac{1}{4} \operatorname{dist}_{E u c l}^{2}(\log (\Sigma), \log (I d))=\frac{1}{4} \int\|\log (\Sigma)\|^{2}
$$

This approach regularizes the full information contained in $\Sigma$, which includes volumetric as well as local anisotropy and orientation changes. This regularizer is thus a natural choice for TBM studies where statistics are performed on $\Sigma$ or a function of it [17.

In elastic-based models, restoring forces increase as deformations become larger, and most models derived under small displacement assumptions can lead to incorrect solutions with tearing or folding in the image medium. This problem can be addressed by using registration equations from fluid mechanics [8]. In this method, the velocity $\boldsymbol{v}$ is the primary variable instead of the deformation field $\boldsymbol{u}$. At each time-step and for each voxel, the velocity field is computed and integrated over time to obtain the final value of $\boldsymbol{u}$.

$$
\boldsymbol{v}(\boldsymbol{x}, t)=\frac{d \boldsymbol{u}(\boldsymbol{x}, t)}{d t}=\frac{\partial \boldsymbol{u}(\boldsymbol{x}, t)}{\partial t}+\boldsymbol{v}(\boldsymbol{x}, t) \cdot \nabla \boldsymbol{u}
$$

We recently implemented a fluid version of the Riemannian elasticity (see equation (11) $)$ in [3] using velocity dependent tensors $\log \left((\nabla \boldsymbol{v}+I d)^{T}(\nabla \boldsymbol{v}+I d)\right)$. A $2 \mathrm{D}$ version of the method was tested in a TBM analysis of the corpus callosum in HIV/AIDS patients [3]. In [4, we developed a 3D version of the algorithm. Accuracy was verified and the algorithm was applied to a small sample of 10 monozygotic and 10 dizygotic twin pairs in order to assess brain volume differences. Here, we further validate this algorithm by performing the first genetic 
study using TBM on a large sample of 92 subjects, 23 pairs of monozygtic (MZ) twins and 23 of same sex dizygotic (DZ) twins. For each of the two groups, resemblances within pairs are assessed by computing the intraclass correlation (ICC) of the determinant of the Jacobian matrices. Significance is shown using color-coded maps of the associated $p$-values. We compare these maps to those obtained after performing the same analysis with a standard fluid registration algorithm [18]. The influence of genes and environmental factors on global lobar volumes were also derived from the two methods using the ACE genetic model, a standard method for variance component estimating in quantitative genetics.

Table 1. Fit of the genetic model for the volumes of the different lobes for the the Riemmanian (R) and the Standard registration (S) methods

\begin{tabular}{|c|c|c|c|c|c|c|c|c|c|}
\hline \multirow{2}{*}{ Lobar region } & \multicolumn{2}{|c|}{$\chi^{2}$} & \multicolumn{2}{c|}{$p$-value } & Lobar region & \multicolumn{2}{|c|}{$\chi^{2}$} & \multicolumn{2}{|c|}{$p$-value } \\
\cline { 2 - 7 } \cline { 7 - 10 } & $\mathrm{R}$ & $\mathrm{S}$ & $\mathrm{R}$ & $\mathrm{S}$ & & $\mathrm{R}$ & $\mathrm{S}$ & $\mathrm{R}$ & $\mathrm{S}$ \\
\hline Frontal lobe (left) & 0.19 & 0.91 & 0.98 & 0.82 & Temporal lobe (left) & 3.33 & 3.73 & 0.34 & 0.29 \\
Frontal lobe (right) & 1.36 & 1.85 & 0.72 & 0.60 & Temporal lobe (right) & 5.43 & 4.70 & 0.14 & 0.19 \\
Frontal lobe (whole) & 0.48 & 1.15 & 0.92 & 0.76 & Temporal lobe (whole) & 3.69 & 4.01 & 0.30 & 0.26 \\
\hline Parietal lobe (left) & 4.40 & 4.25 & 0.22 & 0.24 & Occipital lobe (left) & 12.44 & 10.41 & 0.01 & 0.01 \\
Parietal lobe (right) & 7.74 & 6.01 & 0.05 & 0.11 & Occipital lobe (right) & 2.96 & 2.74 & 0.40 & 0.43 \\
Parietal lobe (whole) & 5.48 & 5.76 & 0.14 & 0.12 & Occipital lobe (whole) & 7.52 & 6.88 & 0.06 & 0.08 \\
\hline
\end{tabular}

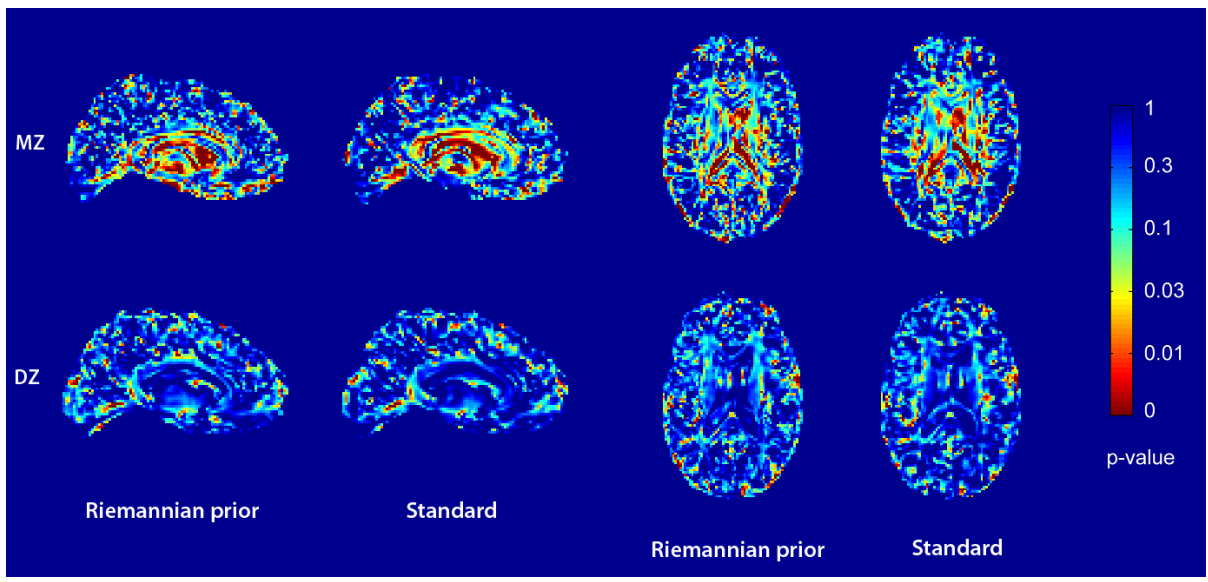

Fig. 1. Sagittal (left) and axial (right) views showing the significance of the intraclass correlation determined within the monozygotic (MZ) and dizygotic (DZ) twin pairs for two different registration algorithms

\section{Method}

\subsection{Data Acquisition and Preprocessing}

We analyzed 3D structural brain MRI scans of 23 monozygotic (MZ) and 23 dizygotic (DZ) same-sex twin pairs (age range: $22-25$ years), as well as that of 


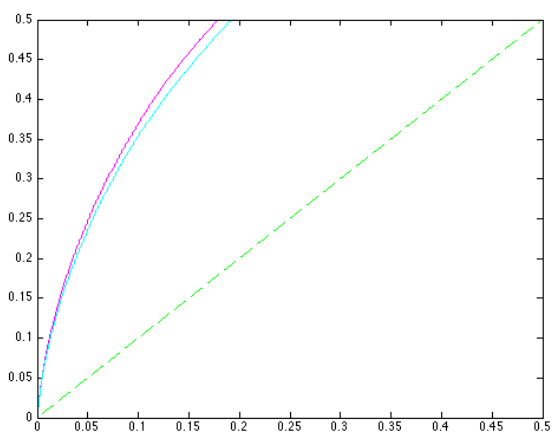

$\mathrm{MZ}$

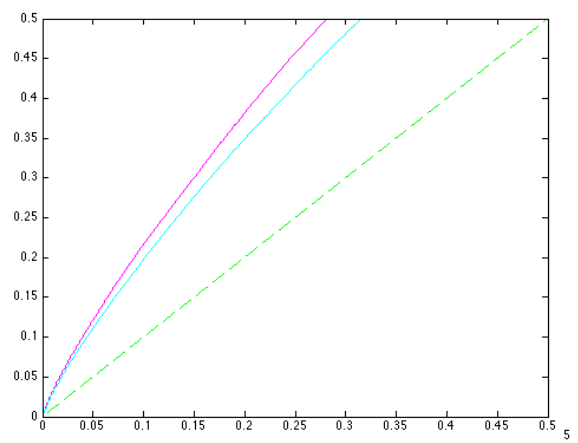

$\mathrm{DZ}$

Fig. 2. Cumulative distribution functions for observed $p$-values vs the corresponding null $p$-value for monozygotic twins (MZ) and dizygotic twins (DZ) for the Riemannian prior (magenta) and standard fluid registration method (cyan). The dotted line shows the expected distribution of $p$-values under the null hypothesis.

an identically scanned healthy subject used as the common target for the fluid registration. $3 D T$-weighted $M P-R A G E$ sequence was used $(T R=2500 \mathrm{~ms}$, $T E=3.83 \mathrm{~ms}, T I=1500 \mathrm{~ms}$, flip angle $=15^{\circ}$, coronal orientation, FOV $230 \mathrm{~mm}$ ) on a 4 Tesla Brucker Medspec whole body scanner (Wesley Hospital, Brisbane, Australia).

Non-brain tissue was deleted from the MRI images with Brain Surface Extractor $(B S E)$ 25] and linearly aligned to the Colin27 template 10. BSE was also used to mask the different lobes of the template, providing regions of interest for volumetric comparisons.

\section{$2.2 \quad$ Registration Method}

Non-linear registration was performed by warping the common template to each individual's 3D scan using the Riemannian fluid algorithm. Averaging images from several study subjects to create a mean template can create a blurry image, and affect the precision of the registration [6]. To avoid this, we choose to use a single subject's scan as a reference [19].

In fluid registration, the transformation is decomposed into several time-steps $\Delta t$. At each of the $\Delta t$, the velocity $v$ is computed and integrated in time to obtain the displacement $\boldsymbol{u}$ at each voxel, according to the equation:

$$
\frac{d \boldsymbol{v}(\boldsymbol{x}, t)}{d t}=\boldsymbol{F}+\nabla \operatorname{Reg}(\boldsymbol{v}, t)
$$

where $F$ denotes the force and Reg the regularizer. Previous fluid algorithms used fluid mechanics equations, such as the Navier-Poisson equation to define the term Reg [8]. For instance in [18, a 3D version of the fluid equation was implemented using a fast filter designed by Gramkow 13. We use this algorithm as the more standard method and compared it to our new Riemannian fluid code. 

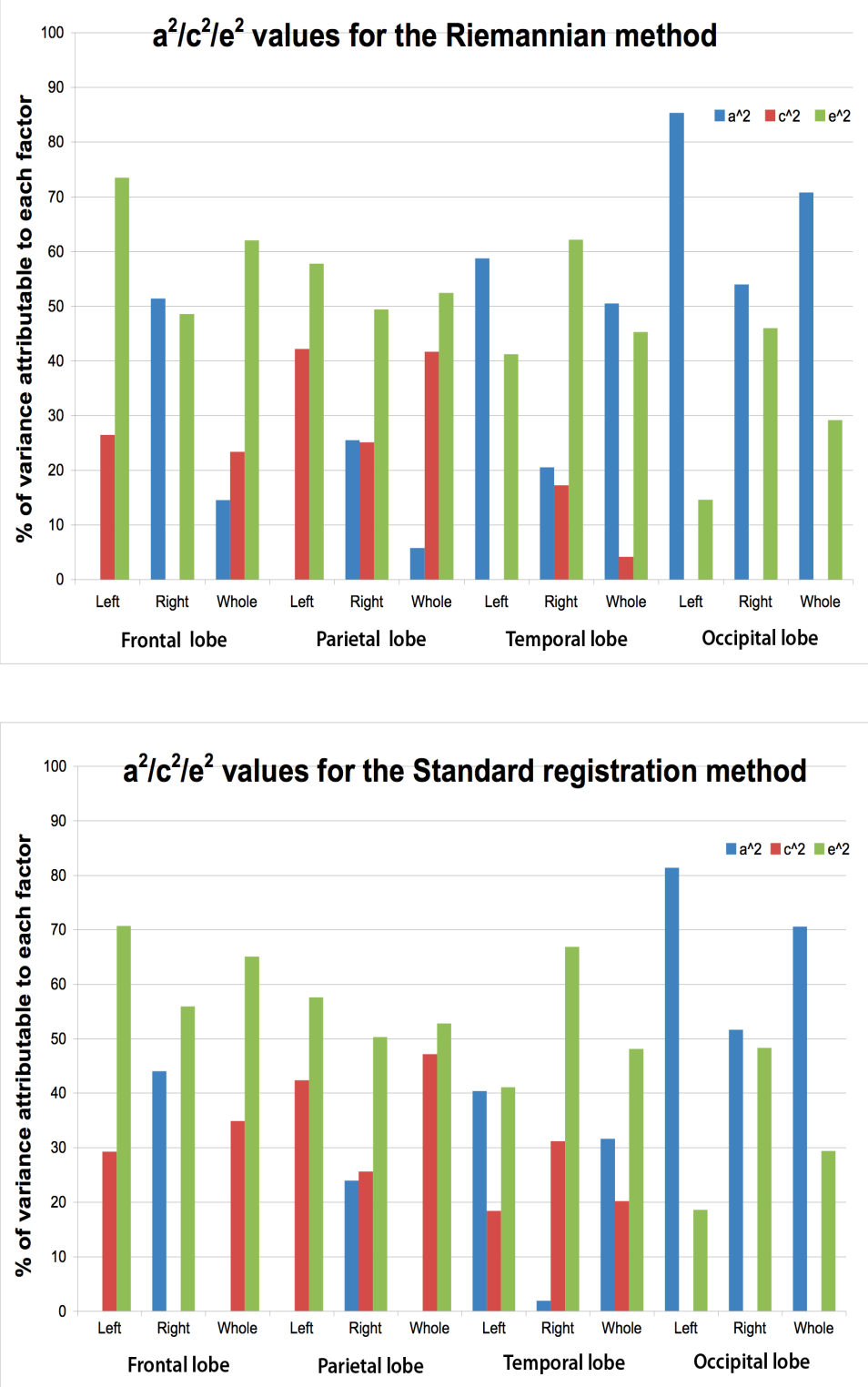

Fig. 3. Variance due to the genetic (A), common environmental (C) and unique environmental (E) factors determined for the Riemannian (top) and the Standard (bottom) fluid registration methods 
The regularizer $R e g_{\text {iso }}$ for the simplified isotropic version of (11) is ([22]):

$$
\operatorname{Reg}_{\text {iso }}(\boldsymbol{u})=\int \frac{\mu}{4} \operatorname{Tr}\left(\left(\log (\Sigma)^{2}\right)+\frac{\lambda}{8} \operatorname{Tr}(\log (\Sigma))^{2}\right.
$$

Here, we integrate this Riemannian prior into the fluid equation, by replacing $\Sigma$ with its fluid equivalent $\log \left((\nabla \boldsymbol{v}+I d)^{T}(\nabla \boldsymbol{v}+I d)\right)$ :

$$
\operatorname{Reg}_{\text {Riem }}(\boldsymbol{v}, t)=\int \frac{\mu}{4} \operatorname{Tr}\left(\log \left((\nabla \boldsymbol{v}+I d)^{T}(\nabla \boldsymbol{v}+I d)\right)^{2}\right)+\frac{\lambda}{8} \operatorname{Tr}\left(\log \left((\nabla \boldsymbol{v}+I d)^{T}(\nabla \boldsymbol{v}+I d)\right)\right)^{2}
$$

The force is derived from an intensity-based similarity measure:

$$
\boldsymbol{F}(\boldsymbol{x}, \boldsymbol{u}(\boldsymbol{x}, t))=-\left.[T(\boldsymbol{x}-\boldsymbol{u}(\boldsymbol{x}, t))-S(\boldsymbol{x})] \nabla T\right|_{\boldsymbol{x}-\boldsymbol{u}(\boldsymbol{x}, t)}
$$

The algorithm is further detailed in 3 .

\subsection{Statistics and Genetic Modeling}

The registration code generates vector fields $\boldsymbol{u}$ at each voxel, for each subject. Jacobian matrices $J=\nabla(x-\boldsymbol{u})$ and their determinants $\operatorname{det} J(\boldsymbol{u})$ are computed from those fields. A value of $\operatorname{det} J(\boldsymbol{u})>1$ indicates a local volumetric expansion of the studied image in comparison to the template whereas $\operatorname{det} J(\boldsymbol{u})<1$ is associated with a local volume shrinkage (see [17]). We created statistical maps from these Jacobians using the Intraclass Correlation Coefficient (ICC) computed independently for the MZ and DZ groups. ICC is a measure of the resemblance between twin pairs, and can be computed for different types of twins ( $\mathrm{MZ}$ and DZ) with different degrees of genetic similarity [24]:

$$
I C C=\sigma_{b}^{2} /\left(\sigma_{b}^{2}+\sigma_{w}^{2}\right) .
$$

$\sigma_{b}^{2}$ is the pooled variance between pairs and $\sigma_{w}^{2}$ is the variance within pairs. A voxelwise permutation test was used to assess the significance of the ICC values, under the null hypothesis of $I C C=0$. The heritability of the different lobe volumes was determined using structural equation models implemented in the genetic modeling program, $\mathrm{Mx}$ (version 1.7.03). In this method, the observed measures arise as a linear function of three latent factors: additive genetic variance $(\mathrm{A})$, common environmental variance $(\mathrm{C})$, and unique environmental variance (E) specific to each individual [20].

\section{Results}

Figure 1 shows statistical maps representing the significance of the ICC at each voxel (under the null hypothesis of no difference) for monozygotic and dizygotic twin pairs for both the Riemannian prior and the standard fluid registration method. Sagittal and axial views are shown. In Figure 2, cumulative distribution functions are plotted for the $p$-values observed for the two algorithms and compared to the $p$-values that would correspond to the null hypothesis (along the line $\mathrm{x}=\mathrm{y}$ ). A significant signal is characterized by a upward large deviation from 
this line. Here, the Riemannian prior gives greater effect sizes in the two cases. Figure 3 displays the percentile measures of $a^{2}, c^{2}$ and $e^{2}$ derived from the ACE model applied to lobar volumes. Results from the Log-Euclidean method and the Standard one are shown and are consistent with each other. The model parameters (goodness-of-fit $\chi^{2}$ and $p$-values) are illustrated in table 1 . This model is adapted to the data for a low $\chi^{2}$ and $p$-value $>0.05$. These conditions are not respected in the left occipital lobe in both cases but are particularly well verified in the frontal lobes.

\section{Conclusion}

We applied a novel Riemannian fluid image registration model to a relatively large twin MRI dataset, and compared regional brain morphometry in individuals who share $100 \%$ and $50 \%$ of their genetic polymorphisms. In computing heritability maps, our Riemannian code largely agreed with the standard fluid registration but gave greater effect sizes, perhaps due to improved regularization and concomitant noise suppression. For both methods, the volumetric correlation was higher, as expected, in the MZ twins than DZ twins, both subcortically and in the corpus callosum. These regions have previously been shown to be heritable (7] 26] 23]). We found that the parietal lobe volumes are relatively highly susceptible to shared environmental influences (exhibiting a high $c^{2}$ variance component), at least compared to occipital lobe volumes, which were predominantly under genetic control (exhibiting a high value for $a^{2}$, the genetic variance component). This is consistent with the neurodevelopmental hypotheses that environmental influences may be relatively greater in brain regions with a more protracted maturational course, while brain regions that mature earliest tend to be more hard-wired according to a common genetic program.

\section{References}

1. Arsigny, V., et al.: Log-Euclidean metrics for fast and simple calculus on diffusion tensors. Mag. Res. Med. 56, 411-421 (2006)

2. Ashburner, J., et al.: A fast diffeomorphic image registration algorithm. Neuro Image 38, 95-113 (2007)

3. Brun, C., et al.: Comparison of Standard and Riemannian fluid registration for Tensor-Based Morphometry in HIV/AIDS. In: Workshop on Statistical Registration, MICCAI, Brisbane, Australia (2007)

4. Brun, C., et al.: A new registration method based on Log-Euclidean tensor metrics and its application to genetic studies. In: ISBI, Paris, France (2008)

5. Cachier, P., et al.: Symmetrization of the Non-Rigid Registration Problem using Inversion-Invariant Energies: Application to Multiple Sclerosis. In: Delp, S.L., DiGoia, A.M., Jaramaz, B. (eds.) MICCAI 2000. LNCS, vol. 1935, pp. 472-481. Springer, Heidelberg (2000)

6. Chiang, M.C., et al.: pattern of brain atrophy in HIV/AIDS visualized using tensorbased morphometry. Neuro image 34, 44-60 (2007) 
7. Chou, Y.Y., et al.: Lateral ventricular surface morphology and volume changes in twins. Neuro image (submitted, 2008)

8. Christensen, G.E., et al.: Deformable templates using large deformation kinematics. IEEE Trans. Image Process 5, 1435-1447 (1996)

9. Chung, M.K., et al.: A Unified Statistical Approach to Deformation-Based Morphometry. Neuro image 14, 595-606 (2006)

10. Collins, D.L., et al.: Design and construction of realistic digital brain phantom. IEEE-TMI 17, 463-468 (1998)

11. Davatzikos, C., et al.: A computerized approach for morphological analysis of the corpus callosum. JCAT 20, 88-97 (1996)

12. Gee, J., et al.: Elastic matching: continuum mechanical and probabilistic analysis. Brain Warping (1998)

13. Gramkow, C.: Registration of 2D and 3D medical images, Master's thesis, Danish Technical University, Copenhagen, Denmark (1996)

14. Grenander, U., et al.: Computational anatomy: An emerging discipline. Quart. of App. Maths. 56, 617-694 (1998)

15. Kochunov, P., et al.: Regional spatial normalization: toward an optimatl target. J. Comput. Assist. Tomogr. 25, 805-816 (2001)

16. Lee, A.D., et al.: 3D Pattern of Brain Abnormalities in Fragile X Syndrome Visualized using Tensor-Based Morphometry. Neuro image 34, 924-938 (2007)

17. Leporé, N., et al.: Generalized Tensor-Based Morphometry of HIV/AIDS using multivariate statistics on deformation tensors. IEEE-TMI 27, 129-141 (2008)

18. Leporé, N., et al.: Tensor based morphometry using fast fluid registration an application to deafness. In: SPIE, San Diego, California, USA (2008)

19. Leporé, N., et al.: Best individual template selection from deformation tensor minimization. In: ISBI, Paris, France (2008)

20. Neale, M.C., et al.: Mx: Statistical modeling. Richmond (1999)

21. Nichols, T.E., Holmes, A.P.: Non parametric permutation tests for functional neuroimaging: a primer with examples. Hum. Brain Map 15, 1-25 (2001)

22. Pennec, X., et al.: Riemannian elasticity: A statistical regularization framework for non-linear registration. In: Duncan, J.S., Gerig, G. (eds.) MICCAI 2005. LNCS, vol. 3750, pp. 943-950. Springer, Heidelberg (2005)

23. Scamvougeras, et al.: Size of the human corpus callosum is genetically determined: An MRI study in mono and dizygotic twins. Neurosci. Lett. 338, 91-94 (2003)

24. Scout, P.E., Fleiss, J.L.: Intraclass correlations: Uses in assessing rater reliability. Psychol. Bull. 2, 420-428 (1979)

25. Shattuck, D.W., Leahy, R.M.: BrainSuite: an automated cortical surface identification tool. Med. Image Anal. 8, 129-141 (2002)

26. Styner, M.: Morphometric analysis of lateral ventricles in schizophrenia and healthy controls, regarding genetic and disease-specific factors. PNAS, 4872-4877 (2005)

27. Thompson, P.M., et al.: Growth Patterns in the Developing Brain Detected By Using Continuum-Mechanical Tensor Maps. Nature 404, 190-193 (2000) 